\title{
ARTIGO ORIGINAL Perfil clínico e sociodemográfico de pacientes com esquizofrenia refratária tratados em um centro terciário
}

\author{
Clinical and sociodemographic profile of patients with \\ refractory schizophrenia treated in a tertiary center
}

Marceli Cezaretto', Ester Franco de Souza Freitas Silva', Ariane Ambrizzi', Victor Eduardo Dutra de Biase', Elissandro de Freitas Silva', Emirene Maria Trevisan Navarro da Cruz', Fabio Aparecido Borghi', Gerardo Maria de Araújo Filho'

\section{RESUMO}

Objetivos: A esquizofrenia está associada a alto grau de incapacidade e importantes déficits neuropsicológicos, sociais e vocacionais. Pesquisas têm sido realizadas com o objetivo de identificar fatores preditivos para refratariedade, a fim de melhorar o tratamento e a qualidade de vida do paciente com esquizofrenia. O presente estudo teve o objetivo de verificar a frequência de pacientes com esquizofrenia refratária acompanhados em serviço terciário, estabelecer o perfil clínico e sociodemográfico e analisar possíveis fatores associados à refratariedade clínica. Métodos: Sessenta e oito pacientes com esquizofrenia foram incluídos no estudo, sendo 36 refratários ao tratamento (52,9\%). Os dados clínicos e sociodemográficos de ambos os grupos foram coletados, analisados e comparados. Um modelo de regressão logística foi elaborado com o objetivo de analisar possíveis fatores associados à refratariedade clínica. Resultados: Entre o grupo refratário, houve maior frequência do sexo masculino $(p=0,03)$, número de antipsicóticos em uso $(p<0,01)$, internações ao longo da vida $(p<0,01)$ e de polifarmácia $(p<0,01)$. Escolaridade, estado civil, história familiar de esquizofrenia e uso de substâncias não foram confirmados como associados à refratariedade. Observou-se atraso temporal entre o estabelecimento da refratariedade clínica e a introdução da clozapina, indicado como o melhor antipsicótico para o tratamento de esquizofrenia refratária. Conclusão: É importante e necessário o desenvolvimento de mais pesquisas a fim de investigar possíveis fatores clínicos e sociodemográficos preditores de refratariedade em pacientes com esquizofrenia, objetivando o início mais precoce de ações terapêuticas.

\section{ABSTRACT}

Objectives: Schizophrenia is associated with a high degree of disability and significant neuropsychological, social and vocational deficits. Studies have been undertaken to identify the predictive factors for refractoriness in order to improve the treatment and quality of life of such patients. The present study aimed to determine the frequency of patients with schizophrenia who are refractory and non-refractory treated in a tertiary center, the clinical and

1 Faculdade de Medicina de São José do Rio Preto (Famerp), Departamento de Psiquiatria e Psicologia Médica.

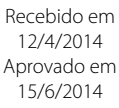

DOI: $10.1590 / 0047-2085000000024$

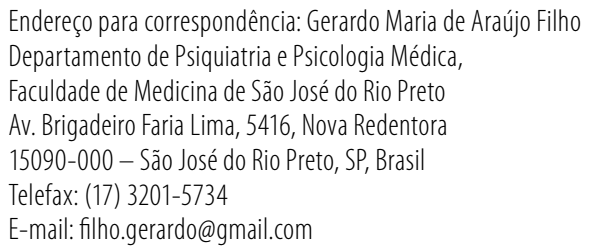




\section{Keywords}

Schizophrenia, psychopathology, associated factors, refractoriness. sociodemographic profile of such population and to analyze the associated factors of clinical refractoriness. Methods: Sixty-eight patients with schizophrenia were included, and 36 were refractory to treatment (52.9\%). Clinical and sociodemographic data of both groups were collected, analyzed and compared. A logistic regression model was elaborated in order to verify possible associations with clinical refractoriness. Results: The refractory group had a higher prevalence of males $(p=0.03)$, antipsychotic use $(p<0.01)$, hospitalizations during lifetime $(p<0.01)$ and polypharmacy $(p<0.01)$. Educational level, marital status, family history of schizophrenia and substance use, were not associated to refractoriness. A delay between the diagnostic of refractoriness and the initiation of clozapine, the best antipsychotic for the treatment of refractory schizophrenia, was also observed. Conclusion: The importance of further research in disclosing possible clinical and socio-demographic predictors of refractoriness in schizophrenia is therefore necessary.

\section{INTRODUÇÃO}

A esquizofrenia é caracterizada pela presença de sintomas positivos (delírios, alucinações e desorganização do pensamento e da conduta), sintomas negativos (embotamento afetivo-volitivo), perdas cognitivas (sobretudo déficit da capacidade de abstração e insight) e sintomas depressivos e ansiosos' ${ }^{1}$. Constitui-se no mais comum dos transtornos psiquiátricos graves, acometendo aproximadamente $20 \mathrm{mi}-$ Ihões de pessoas no mundo e causando importante impacto cognitivo, funcional e afetivo ${ }^{2}$. Dados europeus mostram que menos de $20 \%$ de pessoas com esquizofrenia estavam empregadas no momento do estudo ${ }^{3}$. Nos Estados Unidos, em um seguimento de um ano, 20\% dos portadores de esquizofrenia tornaram-se moradores de rua 4 , o que mantém o empenho dos pesquisadores tanto no campo da neurociência como da clínica na tentativa de melhor compreensão dos seus fenômenos psicopatológicos em busca de tratamento mais efetivo para esses pacientes ${ }^{5}$.

Segundo os critérios de Kane et al. ${ }^{6}$, a esquizofrenia refratária (ER) é caracterizada pela persistência de sintomas positivos moderados a graves, mesmo na vigência de tratamento com doses recomendadas de antipsicóticos por períodos de tempo apropriados (quatro a seis semanas) ${ }^{6}$. Estudos têm sido realizados no sentido de identificar possíveis fatores clínicos e sociodemográficos associados à refratariedade, tais como idade de início da doença, gênero, uso de substâncias psicoativas, número de hospitalizações, doses de medicações e presença de polifarmácia ${ }^{7}$. O objetivo do presente estudo foi o de estabelecer a frequência de pacientes com esquizofrenia não refratária, ER e esquizofrenia super-refratária (ESR) no Ambulatório de Transtornos Psicóticos do Serviço de Psiquiatria do Hospital de Base de São José do Rio Preto, bem como o de verificar possíveis associações de características clínicas e sociodemográficas, tais como gênero, idade, estado civil, escolaridade, início da doença, uso prévio de substâncias psicoativas, histórico familiar e presença de polifarmácia, com a presença de refratariedade clínica ao tratamento antipsicótico.

\section{MÉTODOS}

O presente estudo de corte transversal foi aprovado pelo Comitê de Ética em Pesquisa (CEP) do Hospital de Base da Faculdade de Medicina de São José do Rio Preto (Famerp). Participaram do estudo pacientes adultos de ambos os gêneros que se encontravam em acompanhamento no ambulatório de Transtornos Psicóticos da Famerp há pelo menos seis meses, no período de janeiro de 2012 a janeiro de 2013. Todos os pacientes incluídos no estudo ou os seus responsáveis forneceram o seu consentimento informado.

O diagnóstico de esquizofrenia foi firmado de acordo com critérios da DSM-IV ${ }^{8}$, sendo excluídos pacientes que não concordaram com a participação no estudo, pacientes que apresentavam outras doenças clínicas ou neurológicas graves associadas e pacientes com transtornos psicóticos diferentes de esquizofrenia. Todos os participantes incluídos no estudo responderam a um questionário estruturado para registro dos fatores clínicos, psicopatológicos, familiares e sociodemográficos, entre eles gênero, presença de polifarmácia (uso de três ou mais medicações psicotrópicas), idade de início da doença, escolaridade, estado civil, histórico familiar de esquizofrenia e uso de substâncias psicoativas. Os critérios de Kane et al. ${ }^{6}$ e os algoritmos do IPAP (International Psychopharmacology Algorithm Project) ${ }^{9}$ e do TMAP (The Texas Medication Algorithm Project) ${ }^{10}$ foram utilizados para a determinação da refratariedade clínica da esquizofrenia, separando os pacientes em não refratários e refratários (incluindo nesse último os super-refratários), com objetivo de comparar os dados sociodemográficos na busca por fatores preditivos de refratariedade.

Os resultados dos questionários foram digitados em uma planilha do Microsoft Excel 2007, e a análise estatística foi realizada levando-se em consideração o intervalo de 95\% de confiança $(p<0,05)$, comparando as respostas dos grupos em: não refratários versus refratários e refratários em uso de associações de antipsicóticos versus refratários em uso de clozapina. Para a comparação dos grupos, foram utilizados os testes qui-quadrado $\left(\chi^{2}\right)$ e t de Student, e o teste exato 
de Fisher. Um modelo de regressão logística por meio do método stepwise foi elaborado com o objetivo de identificar possíveis fatores clínicos e/ou sociodemográficos associados à refratariedade clínica.

\section{RESULTADOS}

Dos 147 pacientes cadastrados no ambulatório no período de janeiro de 2012 a janeiro de 2013, apenas 100 eram portadores de esquizofrenia e, desses pacientes, 68 (46,2\%) foram incluídos de acordo com os critérios do estudo. Foram excluídos 79 pacientes, dos quais: 47 eram portadores de outros diagnósticos, 13 possuíam comorbidades neurológicas graves (síndromes demenciais, epilepsia e doenças desmielinizantes) e 17 não completaram o estudo por abandono do tratamento ou por alta para outro serviço. Além disso, um paciente recusou a participação no estudo e um estava internado até o tempo de coleta dos dados do estudo. A frequência de cada um dos diagnósticos encontrados nos pacientes acompanhados está representada na figura 1.

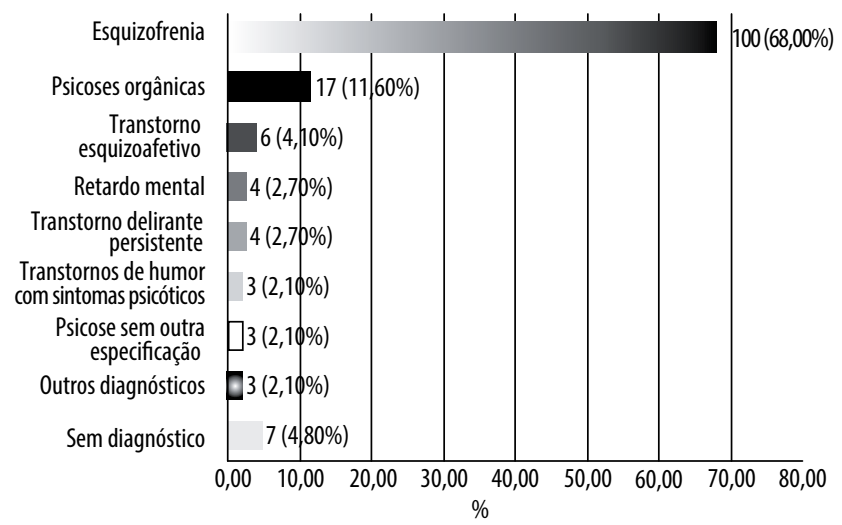

Figura 1. Frequência dos diagnósticos do Ambulatório de Transtornos Psicóticos do Hospital de Base/Famerp.

Dos 68 pacientes com esquizofrenia incluídos no estudo, $52(76,4 \%)$ eram do sexo masculino. Houve maior prevalência do sexo masculino no grupo de pacientes refratários $(83,3 \%$ versus $68,8 \% ; p=0,03)$. A média de idade total foi de $42,03 \pm 13,36$ anos, com intervalo entre 15 e 68 anos. Trinta e dois pacientes $(47,1 \%)$ foram classificados como não refratários ao tratamento e 36 (52,9\%) como refratários e, entre esses, quatro super-refratários. A idade do início da doença foi de 22,1 \pm 10,3 anos para os refratários versus 25,6 $2 \pm 11,6$ anos para os não refratários $(p=0,1)$, e de $21,8 \pm 10,8$ para os super-refratários. Os principais dados clínicos e sociodemográficos dos grupos de pacientes estão expostos na tabela 1.

Acerca do uso de substâncias psicoativas, observou-se que 17 pacientes refratários $(47,2 \%)$ e 13 pacientes não refratários $(40,6 \%)$ experimentaram drogas (incluindo lícitas e ilícitas) antes do diagnóstico de esquizofrenia $(p=0,5)$. Em relação ao uso de cada uma das drogas (álcool, tabaco, maconha, cocaína/crack), não se observaram diferenças significativas na experimentação antes do diagnóstico ou no uso atual ( $p=0,6, p=0,4, p=0,2$ e $p=0,2$, respectivamente). Foi referido uso atual de álcool por dois pacientes do grupo refratário e por nenhum do grupo não refratário $(p=0,1)$, enquanto 10 pacientes do grupo refratário e 6 do grupo não refratário relataram uso atual de tabaco $(p=0,3)$. Ambos os grupos negaram o uso de outras substâncias na atualidade.

Tabela 1. Dados clínicos e sociodemográficos de pacientes com esquizofrenia acompanhados em centro terciário

\begin{tabular}{|c|c|c|c|}
\hline Dado clínico/sociodemográfico & Refratários & Não refratários & $\mathbf{p}$ \\
\hline Número de pacientes & 36 & 32 & - \\
\hline Gênero masculino (\%) & $30(83,3 \%)$ & $22(68,8 \%)$ & $0,03^{*}$ \\
\hline Idade em anos (média $\pm \mathrm{DP}$ ) & $41,8 \pm 11,3$ & $41,0 \pm 10,8$ & 0,5 \\
\hline $\begin{array}{l}\text { Idade de início da doença em anos } \\
\text { (média } \pm \text { DP) }\end{array}$ & $22,1 \pm 10,3$ & $25,9 \pm 11,6$ & 0,1 \\
\hline Estado civil casado (\%) & $6(16,7 \%)$ & $6(18,8 \%)$ & 0,7 \\
\hline História familiar de esquizofrenia (\%) & $18(50,0 \%)$ & $20(62,5 \%)$ & 0,4 \\
\hline $\begin{array}{l}\text { Tentativas de suicídio ao longo da } \\
\text { vida (média } \pm \text { DP) }\end{array}$ & $1,3 \pm 0,4$ & $0,6 \pm 0,2$ & 0,2 \\
\hline $\begin{array}{l}\text { Internações ao longo da vida } \\
\text { (média } \pm \text { DP) }\end{array}$ & $1,8 \pm 0,4$ & $1,0 \pm 0,2$ & $<0,01^{*}$ \\
\hline $\begin{array}{l}\text { Pacientes em uso de }>3 \text { psicotrópicos } \\
(\%)\end{array}$ & $19(52,8 \%)$ & $8(25,0 \%)$ & $<0,01^{*}$ \\
\hline $\begin{array}{l}\text { Pacientes em uso de }>1 \text { antipsicótico } \\
(\%)\end{array}$ & $25(69,4 \%)$ & $2(6,3 \%)$ & $<0,01^{*}$ \\
\hline
\end{tabular}

Os três antipsicóticos mais utilizados entre os pacientes foram: risperidona - 14 refratários $(38,9 \%)$ e 11 não refratários $(34,4 \%)$ (25 pacientes; $p=0,7)$; olanzapina -8 refratários (22,2\%) e 13 não refratários (40,6\%) (21 pacientes; $p=0,1)$; e quetiapina - 10 refratários $(27,8 \%)$ e 4 não refratários $(12,5 \%)$ (14 pacientes; $p=0,1)$. Dos pacientes com esquizofrenia refratária, 69,4\% utilizavam mais de um antipsicótico versus $6,3 \%$ dos não refratários $(p<0,01)$. Os pacientes do grupo refratário utilizavam mais o haloperidol (11 refratários versus 5 não refratários) ( $p=0,03)$ e mais associações de antipsicóticos do que os não refratários. No grupo dos não refratários, observou-se maior frequência de uso de benzodiazepínicos (BZD), e 9 (28,1\%) estavam em uso de algum BZD versus $6(16,7 \%)$ dos refratários $(p=0,2)$. Observou-se que 12 $(37,5 \%)$ pacientes não refratários utilizavam algum tipo de antidepressivo versus $6(16,7 \%)$ dos refratários $(p=0,04)$. Tal diferença, entretanto, ocorreu por causa do uso significativamente maior de inibidores seletivos da recaptação de serotonina (ISRS), entre os pacientes não refratários ( $28,1 \%$ versus 8,3\%; $p=0,02$ ) (Figura 2). Na análise de regressão logística, entretanto, apenas o uso de haloperidol se manteve como significativamente associado ao grupo de pacientes com esquizofrenia refratária. 


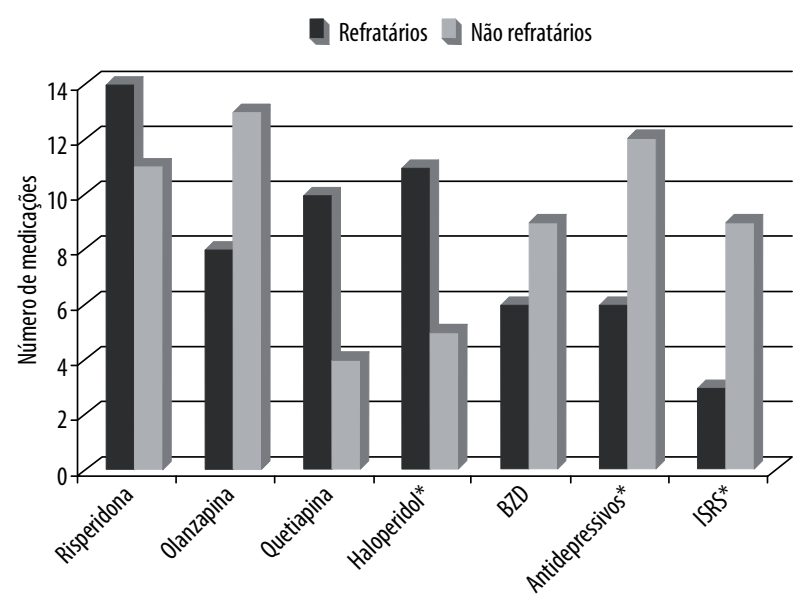

BZD: benzodiazepínicos; ISRS: inibidores seletivos da recaptação de serotonina; ${ }^{*} \mathrm{P}<0,05$.

Figura 2. Frequência das principais medicações utilizadas pelos pacientes com o diagnóstico de esquizofrenia do Ambulatório de Transtornos Psicóticos do Hospital de Base/Famerp.

Quanto ao número de internações ao longo da vida, a média entre o grupo não refratário foi de 1,06 internações versus 1,81 internações entre os refratários $(p<0,01)$. Nos pacientes com ER, 24 (75\%) estavam em uso de associações com antipsicóticos de primeira e segunda geração e 12 em uso de clozapina. A média de idade dos pacientes refratários em uso de clozapina foi de 37,2 anos e a média de idade do início da doença foi de 18 anos. A média de anos do início da doença até a introdução da clozapina foi de 13 anos e a média do tempo do diagnóstico de refratariedade até a introdução da clozapina foi de um ano. Nenhum dos pacientes necessitou de internação após a introdução da clozapina.

Um modelo de regressão logística por meio do método stepwise foi elaborado com o objetivo de identificar possíveis fatores clínicos e/ou sociodemográficos associados à refratariedade clínica. Apesar de terem sido observadas diferenças significativas quanto aos dados clínicos sociodemográficos na comparação dos grupos, os resultados finais ajustados do modelo apontaram que apenas o uso de haloperidol foi significativamente associado ao grupo de pacientes refratários (Tabela 2). Foi realizada outra análise de regressão logística excluindo-se os dados referentes ao tratamento farmacológico, porém nenhum outro fator clínico ou sociodemográfico apresentou associação significante com a refratariedade clínica.

\section{DISCUSSÃO}

O objetivo do presente estudo foi verificar possíveis associações das características clínicas e sociodemográficas com a presença de refratariedade clínica ao tratamento antipsicótico em um centro terciário de tratamento de pacientes com transtornos psicóticos. No referido ambulatório, encontrou-se predomínio de pacientes com diagnóstico de esquizofrenia (68\% dos diagnósticos), destoante dos dados de outros estudos de prevalência de transtornos psicóticos em outros centros de referência ${ }^{10,11}$. Essa prevalência mais elevada em nosso serviço pode ser explicada por ser esse um centro de referência na região, levando ao aumento da demanda relacionada aos casos mais graves. O gênero masculino representou a maioria tanto no grupo dos não refratários quanto no grupo dos refratários, em concordância com outros estudos, que, ao compararem pacientes com ER versus pacientes com esquizofrenia não refratária, também observaram uma frequência significativamente maior de homens ${ }^{12,13}$.

Tabela 2. Fatores de risco associados à presença de refratariedade clínica em pacientes com esquizofrenia acompanhados em centro terciário: resultados finais ajustados de um modelo de regressão logística

\begin{tabular}{lccc}
\hline Fatores de risco & OR ajustada & IC95\% & $\mathbf{P}$ \\
\hline Número de internações & 1 & NA & 0,99 \\
Uso de diazepam & 1 & NA & 0,99 \\
Uso de fluoxetina & 1 & NA & 0,99 \\
Uso de haloperidol & 4,38 & $1,08-2,27$ & $0,03^{*}$ \\
\hline
\end{tabular}

IC: intervalo de confiança; NA: não aplicável; OR: odds ratio; ${ }^{*} p<0,05$.

Dados gerais da literatura apontam que 20\%-30\%, dos pacientes com esquizofrenia são refratários ao tratamento com antipsicóticos. No nosso serviço, entretanto, obtivemos um índice de 52,9\%. Acreditamos que, por ser um ambulatório de referência para casos mais graves, isso tenha representado um viés de seleção. Os pacientes com ER apresentavam uma idade de início da doença entre 17 e 18 anos nos estudos de referência ${ }^{6,7,12,13}$, em contraposição a nossa média, que foi de aproximadamente 22 anos. No entanto, observou-se idade média de aproximadamente 18 anos de início da doença no grupo dos pacientes com ER em uso de clozapina, em concordância com a literatura e reforçando a possível hipótese da idade mais precoce de início da doença como um fator associado à refratariedade. Meltzer et al. ${ }^{12}$ observaram que os pacientes com ER apresentaram idade de início da doença dois anos mais precoce em relação aos pacientes não refratários. Do mesmo modo, Henna et al..$^{13}$ observaram idade de início da doença em torno de 17 anos no grupo com ER e em torno de 20 anos naqueles que apresentavam esquizofrenia não refratária7. Da mesma forma, história prévia de abuso/dependência de substâncias psicoativas tem sido apontada como importante fator associado à refratariedade ${ }^{13}$. O presente estudo, entretanto, não observou essa associação, o que pode ter ocorrido por causa de viés de seleção, uma vez que os pacientes do ambulatório estudado, em sua maioria, são provenientes de outras cidades da região e necessitam de considerável tenacidade para permanecerem em tratamento.

No que se refere à terapêutica, os pacientes com ER, por não responderem a doses usuais das drogas antipsicóticas de 
primeira e de segunda geração, são geralmente tratados tanto com doses mais altas de medicação do que as habitualmente utilizadas como com polifarmácia 4 , o que pode explicar a significância estatística quanto ao número de antipsicóticos utilizados nesses pacientes ${ }^{14,15}$, em concordância, portanto, com os dados encontrados no presente estudo. Além disso, os pacientes não refratários utilizaram maior número de associações com antidepressivos e benzodiazepínicos, o que possivelmente pode ter ocorrido por causa da maior possibilidade do diagnóstico de comorbidades psiquiátricas em pacientes com esquizofrenia quando há controle adequado dos sintomas psicóticos ${ }^{16}$. Por outro lado, observou-se diferença estatisticamente significante entre o uso de ISRS em pacientes não refratários. Sabe-se que os ISRS, de forma geral, atuam como inibidores das subfamílias do citocromo P450, além de aumentarem a ação do ativador do plasminogênio. Tais interações farmacocinéticas e farmacodinâmicas podem aumentar a eficácia dos antipsicóticos e, por conseguinte, produzir maiores resultados clínicos em pacientes com esquizofrenia ${ }^{17}$.

Os antipsicóticos utilizados nesse ambulatório foram prescritos levando-se em consideração o perfil, as comorbidades clínicas e as condições socioeconômicas do paciente, destacando-se a risperidona ou a olanzapina como os mais usados, estando em concordância com as recomendações da literatura ${ }^{18}$. Oliveira ${ }^{20}$ refere que a risperidona e a olanzapina têm sido as drogas mais frequentemente recomendadas como de primeira escolha na esquizofrenia, em decorrência da experiência clínica acumulada ${ }^{20}$. A quetiapina deve ser considerada em presença de resposta parcial ou se houver sintomas extrapiramidais. A clozapina, segundo ensaios clínicos randomizados e metanálises, permanece como melhor opção para os pacientes refratários ao tratamento, sendo o antipsicótico mais eficaz em pacientes com e sem ER ${ }^{18,19}$.

Estudos de custo-eficácia têm mostrado que a clozapina, apesar de seu custo, contribui substancialmente para a redução do risco de recaídas e hospitalizações ${ }^{20}$. Em um estudo comparando clozapina e olanzapina, houve menor necessidade de internação por risco de suicídio no primeiro grupo, redução significativa se considerada a frequência dessa ocorrência em pacientes com esquizofrenia ${ }^{20-22}$. O uso de clozapina é também indicado em pacientes com sintomas extrapiramidais de difícil controle e naqueles portadores de discinesia tardia ${ }^{20-22}$. Apesar dessas evidências, em estudo da literatura realizado com 149 pacientes, a média de atraso teórico para iniciar a clozapina foi de 47,7 meses $^{21,22}$, não correspondendo à realidade do nosso serviço, onde o atraso para a introdução da clozapina foi de apenas um ano. Uma possível explicação para esse fato é a de o referido ambulatório estar associado a uma faculdade de Medicina, constituindo-se em ambiente acadêmico de formação de alunos de graduação e de pós-graduação.

Apesar de terem sido observadas diferenças significativas quanto aos dados clínicos sociodemográficos na compara- ção dos grupos, os resultados finais ajustados do modelo de regressão logística demonstraram que apenas o uso de haloperidol foi significativamente associado ao grupo de pacientes refratários. Entretanto, ao ser realizada outra análise de regressão logística excluindo-se os dados referentes ao tratamento farmacológico, nenhum outro fator clínico ou sociodemográfico apresentou associação significante com a refratariedade clínica. Dessa forma, o resultado obtido poderia estar associado à polifarmácia, presente nos pacientes com ER, e não necessariamente ao uso do haloperidol.

Os resultados do presente estudo devem ser analisados à luz de algumas limitações metodológicas. Em razão de ser um estudo de corte transversal, não houve a possibilidade de acompanhamento longitudinal dos pacientes. Além disso, pelo fato de alguns dados terem sido obtidos por meio de pesquisa de prontuário, e não diretamente com os pacientes, os resultados podem não refletir a exata realidade do seu diagnóstico e/ou do tratamento. Apesar de critérios de inclusão não muito rígidos e compatíveis com outros estudos semelhantes encontrados na literatura ${ }^{6,7,12,13}$, houve considerável perda de pacientes com o diagnóstico de esquizofrenia acompanhados no ambulatório que não foram incluídos na pesquisa (32\%). Por outro lado, o presente estudo corrobora dados da literatura quanto às características clínicas e sociodemográficas de pacientes com ER e não refratária acompanhados em um centro terciário de tratamento no Brasil, contribuindo, dessa forma, para o desenvolvimento de futuros estudos que possam investigar a presença possíveis fatores associados à refratariedade clínica nessa população.

\section{CONCLUSÃO}

Em conclusão, o presente estudo observou que o gênero masculino, a presença de polifarmácia e a idade de início da doença precoce constituíram-se em fatores associados à refratariedade clínica em pacientes com o diagnóstico de esquizofrenia acompanhados em um centro terciário, confirmando dados de literatura. Grau de escolaridade, estado civil, histórico familiar de esquizofrenia e uso de substâncias psicoativas, no entanto, não foram observados como fatores sugestivos de má resposta ao tratamento, refletindo um possível viés de seleção e ressaltando a necessidade da realização de mais pesquisas visando à identificação de fatores biológicos e sociodemográficos preditivos de refratariedade clínica em pacientes com esquizofrenia.

\section{CONTRIBUIÇÕES INDIVIDUAIS}

Marceli Cezaretto - Contribuiu na concepção do projeto e desenho do estudo, coleta de dados, levantamento e revisão bibliográfica, interpretação de dados, redação do artigo 
na íntegra, elaboração das tabelas e gráficos e redação da versão final.

Ester Franco de Souza Freitas da Silva - Contribuiu na concepção e desenho do estudo, coleta de dados, levantamento bibliográfico, análise e interpretação dos dados, elaboração de gráfico, revisão e contribuições críticas na elaboração do artigo.

Ariane Ambrizzi - Contribuiu na concepção do projeto e desenho do estudo, revisão bibliográfica, revisão do artigo e de sua versão final.

Victor Eduardo Dutra de Biase - Contribuiu na concepção do projeto e desenho do estudo, coleta de dados e elaboração de gráfico.

Elissandro de Freitas Silva - Contribuiu no desenho do estudo, análise estatística e interpretação de dados.

Fabio Aparecido Borghi - Participou como orientador na concepção do projeto e desenho do estudo e na aplicação dos questionários estruturados e termos de consentimento aos pacientes.

Emirene Maria Trevisan Navarro da Cruz - Participou como orientadora na concepção do projeto e primeira revisora e revisora intelectual da versão final.

Gerardo Maria de Araújo Filho - Participou como orientador no desenho do estudo e na análise dos dados e revisor intelectual e final.

\section{CONFLITOS DE INTERESSE}

Os autores declaram não haver conflitos de interesse.

\section{REFERÊNCIAS}

1. Miguel EC, Gentil V, Gattaz WF (Eds.). Clínica psiquiátrica. Barueri, SP: Manole; 2011.

2. Gama CS, Souza CM, Lobato MI, Abreu PSB. Relato do uso de clozapina em 56 pacientes atendidos pelo programa de atenção à esquizofrenia refratária da Secretaria de Saúde e do Meio Ambiente do Estado do Rio Grande do Sul. Rev Psiquiatr Rio Gd Sul. 2004;26(1):21-8.

3. Marwaha S, Johnson S, Bebbington P, Stafford M, Angermeyer MC, Brugha T, et al. Rates and correlates of employment in people with schizophrenia in the UK, France and Germany. Br J Psychiatry. 2007;191:30-7.
4. Folsom DP, Hawthorne W, Lindamer L, Gilmer T, Bailey A, Golshan S, et al. Prevalence and risk factors for homelessness and utilization of mental health services among 10,340 patients with serious mental illness in a large public mental health system. Am J Psychiatry. 2005;162:370-6

5. Insel TR. Rethinking schizophrenia. Nature. 2010;468:187-93.

6. Kane J, Honigfeld G, Singer J, Meltzer H. Clozapine for the treatment-resistant schizophrenic: a double-blind comparison with chlorpromazine. Arch Gen Psychiatry. 1988;45(9):789-96.

7. Elkis H, Meltzer HY. Esquizofrenia refratária. Rev Bras Psiquiatr. 2007;29(Supl 2):S41-7.

8. American Psychiatric Association: diagnostic and statistical manual for mental disorders. 4th edition, revised. Washington (DC): American Psychiatric Press; 2004.

9. World Health Organization, International Psychopharmacology Algorithm Project. Disponível em: <http://www.ipap.org >. Acesso em: 27 jul, 2013.

10. Miller AL, Crismon ML, Rush AJ, Chiles J, Kashner TM, Toprac M, et al. The Texas Medication Algorithm Project (TMAP). Schizophr Bull. 2004;30(3):627-47.

11. Porcu M. Prevalência dos transtornos mentais em pacientes atendidos no ambulatório da residência médica de psiquiatria da Universidade Estadual de Maringá. Acta Health Sci. 2007;29(2):145-9.

12. Meltzer HY, Rabinowitz J, Lee MA, Cola PA, Ranjan R, Findling RL, et al. Age at onset and gender of schizophrenic patients in relation to neuroleptic resistance. Am J Psychiatry. 1997:54(4):475-82

13. Henna J, Mello MO, Alves TM, Eizenman IB, Elkis H. Predictors of response and outcome in treatment resistant versus non treatment resistant schizophrenics patients. Schizophr Res. 1999;36(3):281-2.

14. Howes OD, Vergunst F, Gee S, McGuire P, Kapur S, Taylor D. Adherence to treatment guidelines in clinical practice: study of antipsychotic treatment prior to clozapine initiation. $\mathrm{Br} J$ Psychiatry. 2012;201(6):481-5.

15. De Araújo FS, Petribú K, Bastosa 0. Depression in schizophrenia. Rev Bras Psiquiatr. 2002;24(2):86-93

16. Melnik T, Soares BG, Puga M, Atallah NA. Efficacy and safety of atypical antipsychotic drugs (quetiapine, risperidone, aripiprazole and paliperidone) compared with placebo or typical antipsychotic drugs for treating refractory schizophrenia: overview of systematic reviews. Sao Paulo Med J. 2010;128(3):141-66.

17. Geiser F, Conrad R, Imbierowicz K, Meier C, Liedtke R, Klingmüller D, et al. Coagulation activation and fibrinolysis impairment are reduced in patients with anxiety and depression when medicated with serotonergic antidepressants. Psychiatry Clin Neurosci. 2011;65(5):518-25.

18. Englisch S, Zink M. Treatment-resistant schizophrenia: evidence-based strategies. Mens Sana Monogr. 2012;10(1):20-32.

19. Cochrane Schizophrenia Group. Clozapine versus other atypical antipsychotics for schizophrenia. Disponível em: <http://onlinelibrary.wiley.com/o/cocharane/clabout/articles/ SCHIZ/frame.html>. Acesso em: 18 jul, 2013.

20. Oliveira IR. Antipsicóticos atípicos: farmacologia e uso clínico. Rev Bras Psiquiatr. 2000;22(Supl 1):1-8.

21. Honigfeld $G$. The clozapine national registry system: forty years of risk management. J Clin Psychiatry. 1996;14:29-32.

22. Patel MX. Clinician hesitation prior to clozapine initiation: is it justifiable? Br J Psychiatry. 2012:201:425-7. 